\title{
Issues of a combination of ultrasonic doppler velocity measurement with a venturi for multiphase flow metering
}

DOI:

10.2118/164442-MS

Link to publication record in Manchester Research Explorer

\section{Citation for published version (APA):}

Huang, S., Xie, C., Lenn, C., Yang, W., \& Wu, Z. (2013). Issues of a combination of ultrasonic doppler velocity measurement with a venturi for multiphase flow metering. In SPE Middle East Oil and Gas Show and Conference, MEOS, Proceedings/SPE Middle East Oil Gas Show Conf. (Vol. 3, pp. 2101-2109). SPIE.

https://doi.org/10.2118/164442-MS

Published in:

SPE Middle East Oil and Gas Show and Conference, MEOS, Proceedings|SPE Middle East Oil Gas Show Conf.

\section{Citing this paper}

Please note that where the full-text provided on Manchester Research Explorer is the Author Accepted Manuscript or Proof version this may differ from the final Published version. If citing, it is advised that you check and use the publisher's definitive version.

\section{General rights}

Copyright and moral rights for the publications made accessible in the Research Explorer are retained by the authors and/or other copyright owners and it is a condition of accessing publications that users recognise and abide by the legal requirements associated with these rights.

\section{Takedown policy}

If you believe that this document breaches copyright please refer to the University of Manchester's Takedown Procedures [http://man.ac.uk/04Y6Bo] or contact uml.scholarlycommunications@manchester.ac.uk providing relevant details, so we can investigate your claim.

\section{OPEN ACCESS}




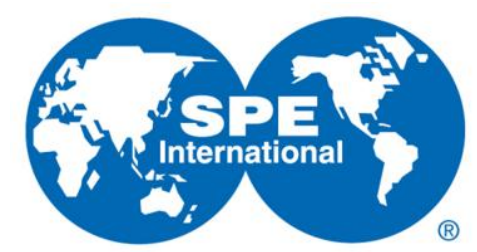

\title{
Issues of a Combination of Ultrasonic Doppler Velocity Measurement with a Venturi for Multiphase Flow Metering
}

\author{
Huang, S., Xie, C. and Lenn, C., Schlumberger \\ Yang, W. and Wu, Z., University of Manchester
}

Copyright 2013, Society of Petroleum Engineers

This paper was prepared for presentation at the SPE Middle East Oil and Gas Show and Exhibition held in Manama, Bahrain, 10-13 March 2013.

This paper was selected for presentation by an SPE program committee following review of information contained in an abstract submitted by the author(s). Contents of the paper have not been reviewed by the Society of Petroleum Engineers and are subject to correction by the author(s). The material does not necessarily reflect any position of the Society of Petroleum Engineers, its officers, or members. Electronic reproduction, distribution, or storage of any part of this paper without the written consent of the Society of Petroleum Engineers is prohibited. Permission to reproduce in print is restricted to an abstract of not more than 300 words; illustrations may not be copied. The abstract must contain conspicuous acknowledgment of SPE copyright.

\begin{abstract}
Multiphase flowmeters (MPFMs) available today are mostly based on a combination of differential pressure measurement, provided by at least one flow constriction, such as a Venturi device, with phase holdup measurement based on nuclear techniques, electromagnetic techniques, or both. This paper investigates the issues of combining the ultrasonic Doppler velocity measurement with the differential pressure measurement for multiphase flow metering. The intention is to measure at least one "useful" velocity of the flow, such as the bulk liquid velocity or the homogeneous mixture velocity, which can then be combined with a measured differential pressure across a "constriction" device, such as a Venturi, to derive parameters needed to calculate liquid and gas flow rates. An ultrasonic Doppler velocity sensor has been tested in combination with a Venturi under multiphase flow conditions. The results show that, due to the effect of gas bubbles in the liquid phase, the interrogation depth of the ultrasonic wave is often limited to a shallow liquid region near the pipe wall. As a result, the ultrasonic Doppler sensor cannot reliably measure the bulk liquid velocity, or the homogeneous velocity. It is generally difficult to interpret the measured Doppler velocity and to derive the flow rates of a multiphase flow without employing an elaborate flow model. This work was part of a project cofunded by the UK Technology Strategy Board ${ }^{1}$, with industry partners The University of Manchester, Schlumberger Gould Research, and TUV-NEL.
\end{abstract}

\section{Introduction}

Many multiphase flow meters utilize differential pressure measurement combined with a mixture density or gas holdup measurement [1,2]. A typical example is a dual-energy gamma-ray system that measures the density and the water-liquidratio (WLR) of multiphase mixture at the throat of a Venturi that provides the differential pressure measurement [3]. The measured density of the mixture (hence gas/liquid holdup) and WLR are then combined with the differential pressure across the Venturi to derive the flow rates of the three individual phases: oil, gas, and water. Other approaches used in MPFMs include combining differential pressure measurement with velocity measurement to derive the liquid and gas flow rate [1]. A recent MPFM [4] utilizes differential pressures across two flow constrictions and an ultrasonic Doppler velocity measurement to obtain the flow rates of gas, oil and water. It was claimed that the ultrasonic Doppler sensor could measure the velocity of a homogeneous multiphase flow, even though no flow homogenization was used in the MPFM. As remarked in [2], the direct velocity measurement technique widely used in MPFMs today is based on cross-correlation, which tends to measure the velocity of large and distinctive dispersed-phase flow structures, such as gas slugs. As a result, the velocity measurement based on cross correlation is not necessarily useful because it is difficult to find consistent correlations between a slug velocity and the "useful" velocities, such as the velocity of homogeneous mixture, which is defined as the total volumetric flow rate of all the phases divided by the cross-section of the pipe bore, or the velocity of bulk liquid phase.

\footnotetext{
${ }^{1}$ The Technology Strategy Board is a business-led governmental body that creates economic growth by promoting the UK's role as a global leader in innovation. Sponsored by the Department for Business, Innovation, and Skills (BIS), it brings together business, research, and the public sector, accelerating the development of innovative products and services to meet market needs, tackle major societal challenges, and help build the future economy.
} 
In this paper, we first explore how a relevant velocity measurement can be combined with a differential pressure measured across a Venturi to derive parameters, such as the density of mixture. Then we describe an experimental investigation of a flow velocity sensor based on the range-gated ultrasonic Doppler technique. We are particularly interested in how the gas bubbles, which are likely to be present in the liquid phase, affect the propagation of ultrasonic waves. Our data shows that it is difficult to make reliable measurements of "useful" velocities because gas entrainment limits the depth of investigation of the ultrasonic technique.

\section{Concept}

The basic approach to deriving flow rates of liquid and gas phases in a multiphase flow by combining Venturi $\Delta P$ with a measured velocity of bulk liquid phase is described below. The relationship between the total mass flow rate, $Q_{\text {total }}$, and the differential pressure across the Venturi, $P_{V}$, is

$$
Q_{\text {total }}=\frac{C_{d} A_{T}}{\sqrt{1-\beta^{4}}} \sqrt{2 \Delta P_{V} \rho_{\text {mix }}},
$$

where $\beta$ is the throat/inlet diameter ratio of the Venturi, $A_{T}$ is the throat cross-sectional area, $C_{d}$ is the discharge coefficient that can be determined via appropriate calibration and $\rho_{\text {mix }}$ is the density of the multiphase mixture at the Venturi throat.

Assuming that the targeted applications have relatively low operating pressures ( $<30$ bar) and the gas-volume-fraction $(\mathrm{GVF})$ is less than $90 \%$, then the total mass flow rate can be approximately represented by the mass flow rate of the liquid phase, $Q_{\text {liq }}$ alone, i.e.

$$
Q_{\text {total }} \approx Q_{\text {liq }}=\rho_{\text {liq }} \alpha_{\text {liq }} V_{\text {liq }} A_{T},
$$

where $\alpha_{l i q}, \rho_{l i q}$ and $V_{l i q}$ are the holdup, density and velocity of the liquid phase, respectively.

Combining Eqs. (1) and (2) yields

$$
\Delta P_{V} \approx \frac{1-\beta^{4}}{2 C_{d}^{2}} \alpha_{l i q} \rho_{l i q} V_{l i q}^{2}
$$

From Eq (3), we can obtain an estimate of the liquid holdup from the Venturi $\Delta \mathrm{P}$ and the velocity of liquid phase, if such a velocity can be measured. The volumetric liquid flow rate can then be estimated by combining the measured velocity of the liquid phase with the derived liquid holdup. The knowledge of the liquid holdup also leads to the determination of the gas holdup. By using a suitable gas-liquid slip model [5], the GVF and the gas flow rate at the measurement section can also be estimated.

A different approach is to measure the homogeneous velocity of the gas/liquid mixture, $V_{h}$. Assuming that at the throat of Venturi the gas and liquid are mixed well together and the slip velocity between the bulk gas and the bulk liquid is small compared with $V_{h}$, then the total mass flow rate can be approximately expressed as

$$
Q_{\text {total }} \approx V_{h} A_{T} \rho_{\text {mix }}
$$

By such an approximation, the following approximate relationship can be derived from Eq (1):

$$
V_{h} \approx \frac{C_{d}}{\sqrt{1-\beta^{4}}} \sqrt{\frac{2 \Delta P_{V}}{\rho_{\text {mix }}}}
$$

Eq (5) enables the derivation of $\rho_{\text {mix }}$ from $\Delta P_{v}$ and $V_{h}$, if the latter can be measured. Once we have the mixture density and a measured WLR, the liquid holdup can be derived. A slip law can then be used to obtain the GVF from the holdup. Finally the volumetric flow rates of gas and liquid phase can be obtained from the measured $V_{h}$ and a derived GVF.

Both Eqs. (3) and (5) show that the variables to be derived, $\rho_{\text {mix }}$ and $\alpha_{\text {liq }}$, are a function of $V_{\text {meas }}^{2}$, where $V_{\text {meas }}$ represents either the measured homogeneous velocity or bulk liquid velocity. By differentiating Eq (3) or (5), the relative error of the derived parameter is:

$$
\frac{\Delta \alpha_{l i q}}{\alpha_{l i q}}=-2 \frac{\Delta V_{l i q}}{V_{l i q}} \quad \text { (6) or } \quad \frac{\Delta \rho_{\text {mix }}}{\rho_{\text {mix }}}=-2 \frac{\Delta V_{h}}{V_{h}}
$$

Eq (6) or (7) shows the importance of an accurate velocity measurement to the approaches described above because a $5 \%$ relative error in $V_{\text {meas }}$ will result in a $10 \%$ relative error in the derived parameter. To put things into context, a nuclear-based system can directly measure parameters such as $\rho_{\text {mix }}$ with an accuracy of a few percent. For a flowmeter with a $\pm 10 \%$ error for liquid flow rate measurement, the required homogeneous velocity or the liquid velocity should be measured within at least $\pm 5 \%$ of the reading. 


\section{Experimental system}

Pulsed-wave ultrasonic Doppler measurement method

There are many reports in the literature regarding applications of ultrasonic Doppler techniques for oilfield multiphase flow measurement, both down-hole and surface [6-8]. Among these, the pulsed-wave (PW) ultrasonic Doppler method is often used. Compared with the continuous-wave method, the PW method allows a range-gating technique to be applied to the ultrasonic echo signal, producing velocity- and echo-energy profiles versus the depth of interrogation [9]. Multiphase flows with velocity distributions over the cross section of a pipe are often unpredictable. Therefore, being able to provide a velocity profile measurement is a main advantage. Further, the echo-energy profiles are useful in identifying the main interfaces between different fluid phases [11].

The basic principle for the PW ultrasonic Doppler system used in our investigation is shown in Fig. 1. In the measurement mode, a series of ultrasonic pulses (sinusoidal bursts of $\mathrm{MHz}$ frequencies repeated at a pulse repetition frequency $\left[f_{p r f}\right]$ of typically around $\mathrm{kHz}$ range) are emitted from the transducer probes into a flow at an appropriate angle, $\alpha_{2}$. After the ultrasonic pulse enters the flow, echo signals are generated by the entrained reflectors in the continuous liquid phase, such as liquid droplets (if more than one liquid phase is involved), gas bubbles, or both. These echo signals are routed through a transmitting/ receiving (T/R) switch to the input of the receiver. Following the preamplification and ADC stages, the analog signals are converted into a digital form, which is further processed digitally through a demodulator and a Doppler signal processor, where a pulse-echo record is divided into many small time-delay intervals (see Fig. 1).

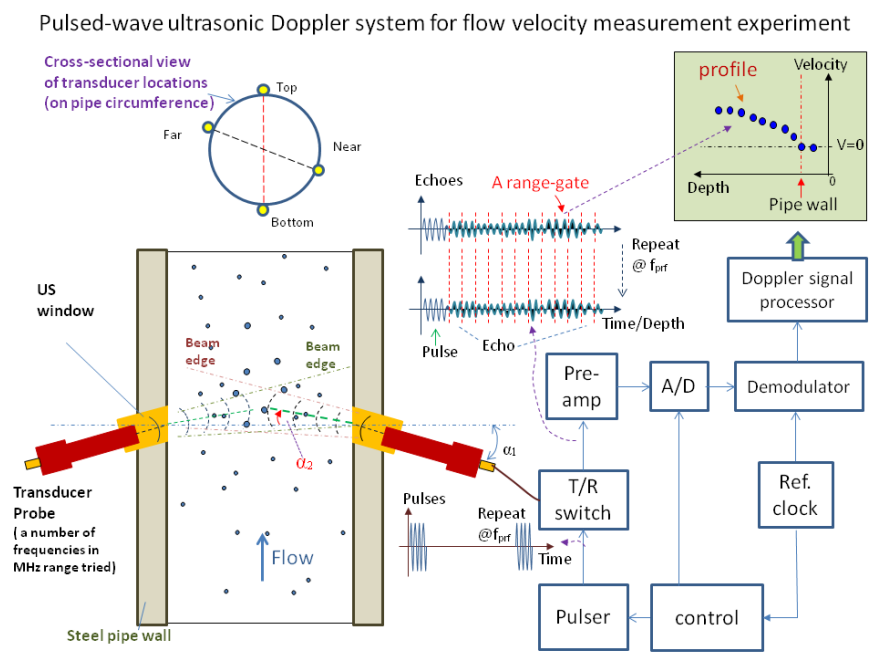

Fig. 1-Experimental setup and operating principle of PW ultrasonic Doppler measurement system

If the speed of sound in the liquid phase is known (which is the case in our test), then the echo delay time can be converted into a depth of interrogation, according to:

$$
d=0.5 t c_{\text {mix }} \cos \left(\alpha_{2}\right)
$$

where $t$ is the delay time and $c_{m i x}$ is the speed of sound of the oil/water mixture used in our test. It can be obtained, for example, from the linear interpolation of the speed of sound values for pure oil and water and the reference WLR:

$$
c_{\text {mix }}=c_{\text {oil }}(1-W L R)+c_{\text {water }} W L R
$$

Using Eq (8), the time intervals can be converted into the depth increments, which are referred to as the range gates. Typically, a number of consecutive pulse-echo records are used together in the Doppler signal processing, in which the echo signals from the same range gate (i.e., the depth) across several records are grouped together to produce one average Doppler frequency shift (and energy level) estimation, $\Delta f_{i}$ (and $E_{i}$ ) for the $i^{\text {th }}$ range gate. Such a frequency shift can then be converted to a local flow velocity by using the Doppler formula:

$$
V_{i}=\frac{c_{m i x}}{2 \cos \left(90^{\circ}-\alpha_{2}\right)} \frac{\Delta f_{i}}{f_{e}}
$$

where $f_{e}$ is the emitting frequency of the transducer probe. Doing this for all of the range gates produces a flow velocity versus the depth profile (and also an echo energy profile) across the flow, as shown in Fig. 1. With an appropriate Doppler frequency-estimation algorithm [9], these flow profiles can be generated in real time, typically with an update rate of a few hundred frames per seconds.

\section{Measurement system setup, transducers}

Fig. 1 also shows the experimental setup where several ultrasonic narrowband transducer probes are mounted around a flow-conveying pipe of 2-in [50 mm] inner diameter, which is the throat section of a vertically installed Venturi. A total of four transducers, working in the $\mathrm{MHz}$ range, are mounted in such a way with their circumferential locations shown in the upper left part of Fig. 1. Ultrasound-transparent windows facilitate the cross-pipe transmission of acoustic energy from the transducer probes into the liquid phase in the pipe. The thickness of the window provides a sufficient separation between the probe and the flow, ensuring that the near field of the ultrasonic beam ends before reaching the flow. The mounting holes in the windows also enable an oblique beam incidence angle $\left(\alpha_{1}\right)$ to be set with respect to the radial direction of the pipe. At the 
window/fluid interface, the pulsed ultrasonic beam enters the flow (see the broken green line in Fig. 1) at a refraction angle $\left(\alpha_{2}\right)$ determined by Snell's Law:

$$
\frac{\sin \alpha_{1}}{c_{1}}=\frac{\sin \alpha_{2}}{c_{2}}
$$

where $c_{1}$ and $c_{2}$ are the speed of sound in the window and in the fluid respectively, and $\alpha_{2}$ is the refraction angle in the fluid.

In this experimental work, we used four independent data acquisition channels to perform simultaneous Doppler measurements with the four transducers. In each channel the measured analog echo signal is digitized via a high-speed ADC, and the subsequent processing, such as demodulation and Doppler frequency estimation, is done digitally. The details of the ultrasonic Doppler signal processing chain and relevant algorithms for producing the estimated frequency shift and signalenergy profiles can be found in [9].

\section{Calculating velocity of bulk liquid from Doppler profiles}

The steps of calculating the liquid-phase flow velocity from the measured Doppler velocity and energy profiles are as follows:

- From each instantaneous Doppler signal-energy profile, the signal intensity is analysed to find the end of the liquid region, assuming a concentric annular gas/liquid distribution;

- The section of the velocity profile between the inner pipe wall and the end of the liquid region identified in step 1 is integrated;

- The average velocity value $V\left(t_{j}\right)$ is calculated in this way from each of four instantaneous velocity/energy profiles, and a time average liquid-phase velocity is obtained by averaging the time series $V(t)$ over the acquisition period.

To demonstrate the process, we tested a vertical three-phase gas/oil/water flow with a total liquid rate $\mathrm{Q}_{\text {liq }}=30 \mathrm{~m}^{3} / \mathrm{hr}$, $\mathrm{GVF}=0.25$, and WLR=0.9. Fig. 2 shows the time series of the measured Doppler velocity (averaged across the ultrasound interrogation depth) and the measured apparent thickness of liquid layer over a 10-s period for this flow condition. Obviously, this is a slug flow, alternating between liquid-continuous sections (with some gas bubbles), where the measured thickness of liquid layer can sometimes reach the pipe center (lower plot in Fig. 2), and gas slugs, where only a thin liquid annulus on the pipe wall is measured.

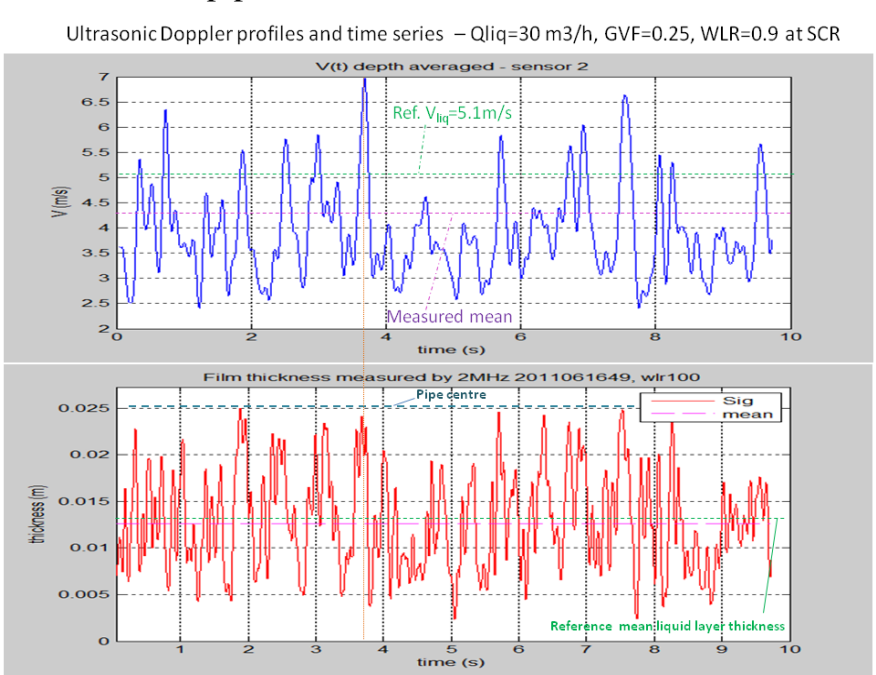

Fig. 2-Time series of measured liquid layer velocity through weighted integration from the inner pipe wall to the apparent gas/liquid interface (upper plot) and measured thickness of liquid layer of gas/oil/water flow, showing a slug flow regime (lower plot). Note that a thick liquid layer usually correlates with a velocity peak, as marked by the orange vertical line.

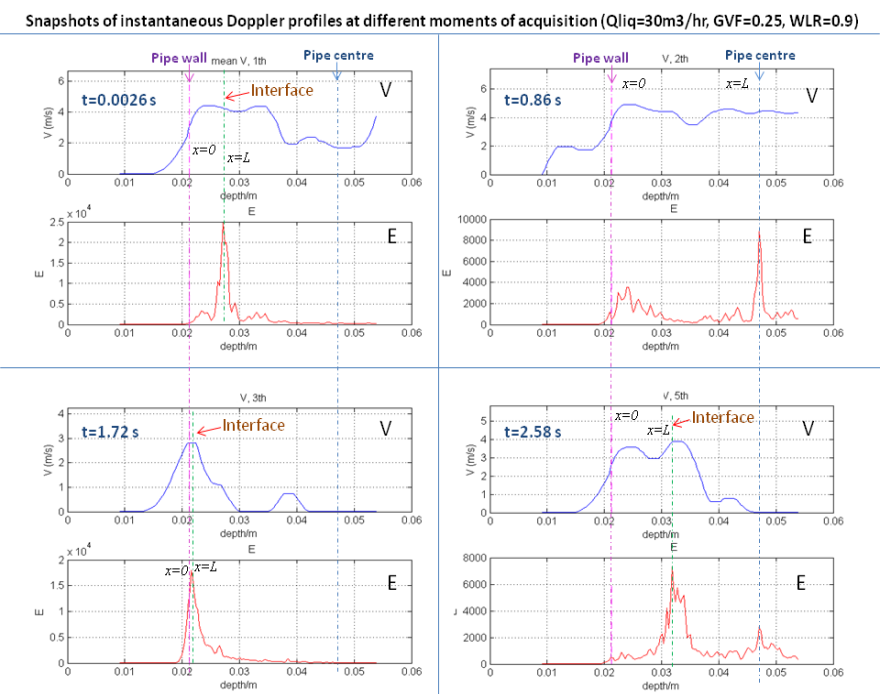

Fig. 3 - Instantaneous Doppler velocity ('V') and energy ('E') profiles captured at four different time points, $\mathrm{t}=0.0026,0.86,1.72$, and $2.58 \mathrm{~s}$, in a gas/oil/water slug flow with $\mathrm{Q}_{\text {liq }}=30 \mathrm{~m}^{3} / \mathrm{hr}, \mathrm{GVF}=0.25$, and $\mathrm{WLR}=0.9$, with inner pipe-wall, detected gas/liquid interface and pipe center marked by vertical lines.

Fig. 3 shows some snapshots of the instantaneous velocity and signal-energy profiles produced by the ultrasonic Doppler measurement system for the same flow condition, where the non-zero velocity profile section within the inner wall appears to have been caused by the weak residual signals from the previous pulse. At $t=0.0026 \mathrm{~s}$ (the upper left plot), the processing algorithm determines the gas/liquid interface location from the maximum peak of the signal-energy (E) profile. The liquid region between $x=0$ (the inner pipe wall) and $x=L$ (the end of the liquid region) is defined for the integration calculation (step 2). The location of the E peak, between the inner wall and the pipe center, suggests that the body of a gas slug is detected. At 
$t=0.86 \mathrm{~s}$ (upper right), the maximum $\mathrm{E}$ peak overlaps with the location of the center of the pipe, suggesting that this is a liquid-continuous section with so few gas bubbles that the pulse from the probe on the opposite side of the pipe (see Fig. 1) can be received directly. At $t=1.72 \mathrm{~s}$ (lower left), the E peak is very close to the wall, indicating very limited interrogation depth caused either by a large diameter gas slug or by gas bubbles located near the wall. This situation leads to a smaller measured velocity than in the first two cases. At $t=2.58 \mathrm{~s}$ (lower right), the maximum E peak is detected at $x=L$ while a small peak also appears at the center of the pipe. This could be a liquid-continuous section with sufficient gas bubbles to produce the maximum E peak, allowing attenuated pulse energy from the opposite probe to be received. Whatever the complexity of the distribution, the processing algorithm simply integrates the velocity profile from $x=0$ to what it identifies as the apparent $x=L$. Therefore, the Doppler velocity measurement based on the method described in this section may potentially be affected by the presence of gas bubbles in the liquid phase. If the bubbles near the pipe wall produce the largest echo, then the integration will only be done over a limited depth into the liquid phase, producing a nonrepresentative measurement of the average velocity of the liquid. This is a fundamental limitation of the ultrasonic measurement that is difficult to avoid.

\section{Test apparatus setup on TUV-NEL's multiphase flow loop}

The test apparatus setup on TUV-NEL's multiphase flow loop is for vertical upward flow with a blind-tee inlet. As shown in Fig. 4, the instrumented Venturi prototype forms part of the vertical upleg of the upside-down U-shaped installation. However, to accommodate different types of velocity and (potentially) holdup sensors, including the ultrasonic Doppler probes, an extended Venturi throat section was used. The locations of the differential pressure and ultrasonic measurement are also shown in Fig. 4.

\section{Test matrix and test procedures}

The test matrix is shown in the form of combinations of gas and liquid flow rates in Fig. 5 for TUV-NEL tests, where different WLR values are marked by different color legends and different GVF ranges divided by different colored lines. The non-zero GVF range spans mostly from 0.25 to 0.95 , although for low liquid flow rates $\left(<20 \mathrm{~m}^{3} / \mathrm{hr}\right)$, the lower end of the GVF range starts at about 0.4 due to the lowest gas flow rate available from TUV-NEL's multiphase flow loop. The following fluids were used for the test:

- Oil: a mixture of crude oil with light components removed and replaced by kerosene to create the original viscosity and density, which are about $10.4 \mathrm{cP}$ and $0.853 \mathrm{~kg} / \mathrm{L}$, respectively, at $104 \mathrm{degF}$ [40 degC].

- Water: an aqueous solution of magnesium sulphate of concentration $80 \mathrm{~g} / \mathrm{L}$ (based on $\mathrm{MgSO}_{4} \cdot 7 \mathrm{H}_{2} \mathrm{O}$ ), which gives a density of $1.034 \mathrm{~kg} / \mathrm{L}$ and a viscosity of $0.86 \mathrm{cP}$ at $104 \mathrm{degF}$ [40 degC].

- Gas: nitrogen.

\section{Doppler test setup at NEL - (21-25 March 2011)}
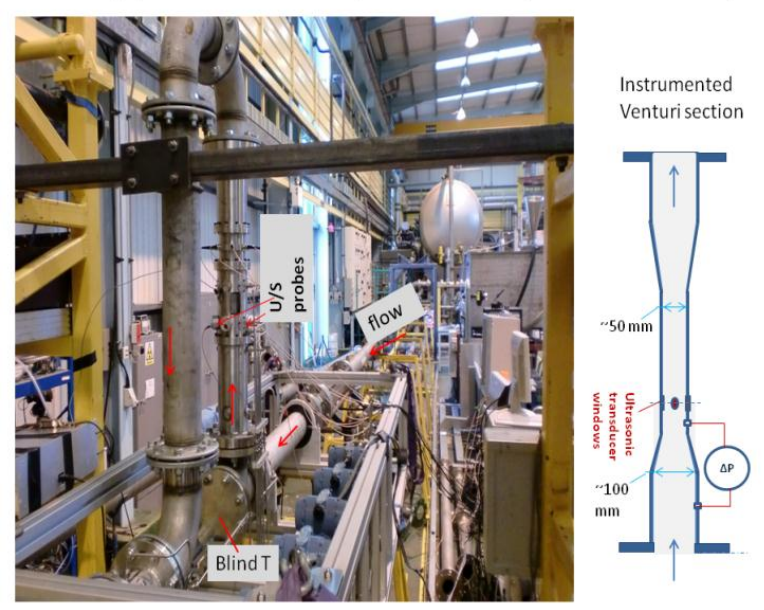

Fig. 4-Ten-bar pressure-rated flowmeter test setup with a blind-tee inlet at TUV-NEL; the upleg has the instrumented Venturi throat with ultrasonic Doppler probes.

During the test, the flow loop pressure was controlled at around 5 bar (gauge) and temperature at about $104 \operatorname{degF}$ [40 $\operatorname{deg}$ C]. In a typical test sequence, a WLR was chosen first and then a fixed liquid flow rate selected. The gas flow rate varied to produce different GVF conditions. Such a sequence was repeated for all the liquid flow rates and WLR values. For each test point, data acquisition always started at least about $2 \mathrm{~min}$ after changing the water flow rates, oil flow rates, or both, 
allowing sufficient settling time of the flow. An acquisition time of 40 seconds was used for each test point, which was sufficiently long for averaging out the fluctuation of the multiphase flows. The analog signals from the two measurement systems (i.e., the differential pressure and the ultrasonic Doppler) were digitized and stored as data files using appropriate data acquisition systems. Further signal processing and parameter calculations were performed offline.

\section{Results}

Velocity measurement of oil/water two-phase flows

In the first test, a few oil/water flows were run to calibrate the PW ultrasonic Doppler velocity measurement system. With zero gas flow rate and liquid flow velocities of greater than $5 \mathrm{~m} / \mathrm{s}$ at the Venturi throat, the liquid-only flow can be regarded as a homogeneous oil and water mixture with negligible slip between the two liquid components. Under such circumstances, the back-scattered echo energies are generated from the discrete oil or water droplets in the flow. By assuming nearly flat velocity profiles across such turbulent flows, the measured Doppler velocities should be close to the reference homogeneous velocity of the oil/water two-phase flows. Therefore, such a test is regarded as well-controlled calibration.

Fig. 6 shows the calibration results, which are obtained at a fixed liquid flow rate albeit with a variable WLR. The measured average flow velocities and the reference liquid (homogeneous) velocities are plotted versus the WLR in the upper plot. The variance of the measured velocity and the relative strength of the back-scattered echo energy (with respect to the maximum strength at WLR=0.5) are shown in the lower plot. Comparing the measured velocity values in the upper plot with the relative strength of the back-scattered Doppler signal in the lower plot (green stars), it is obvious that there is a correlation between low signal strength and inaccurate velocity measurement. Low signal strength is typically associated with low droplet concentration $(<10 \%)$ of the discrete liquid phase. Although for the flow condition represented by the cyan circle, the water flow rate through the water pump was actually set to zero, the contamination of the oil phase by water was so serious that there was $18 \%$ water already in the oil stored in the separator. Because of the difficulty in separation of water from oil, water must have existed in the

Ultrasonic - Measured and Ref. $V_{\text {liquid }}$ versus WLR at NEL
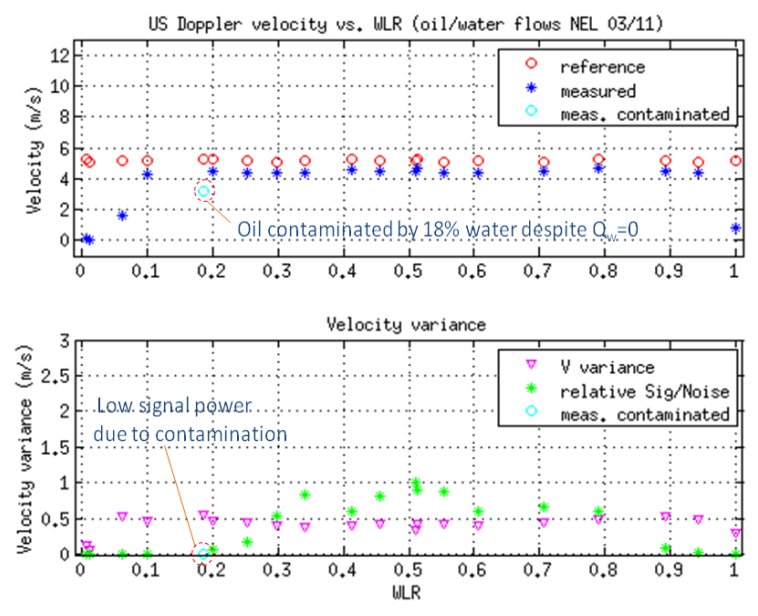

Fig. 6-Calibrating the ultrasonic Doppler system with oil/water two-phase flows at a fixed total liquid flow rate but different WLR values. Measured average flow velocities (upper plot); variance of velocity-measurement magenta triangles and relative signal strength green stars (lower plot), both plotted against the reference WLR. form of very fine droplets, much smaller than the ultrasonic wavelength and, therefore, unable to produce sufficient backscattered signal energy.

Even for the successful velocity measurements within the WLR range of 0.1 to 0.95 , there is a consistent underestimation of about $15 \%$ by the ultrasonic Doppler sensor. This apparent systematic error is consistent with previous investigation and was explained by a beam spread effect in [10]. In the subsequent velocity measurements, this $15 \%$ systematic error is corrected before the results are presented. For oil/water two-phase flows within the WLR range of 0.1 to 0.95 , the measurement uncertainties of the ultrasonic Doppler system are well within $\pm 10 \%$ of the reading, after the systematic error correction.

\section{Reference velocities of gas/oil/water three-phase flows}

For the three-phase flow test conditions, two reference velocities were mainly used to validate the ultrasonic Doppler velocity measurement system. The reference homogeneous velocity can be readily obtained from the reference flow rates of the three phases measured by flowmeters on the single phase feed lines of the flow loop. The reference bulk liquid velocity can be derived by combining the measured Venturi $\Delta \mathrm{P}$ with the known reference flow rates of the three phases, using Eq (1).

Fig. 7 shows the derived velocity of the reference liquid phase plotted versus the reference homogeneous velocity for the TUV-NEL test. With all the points located below or on the 1-to-1 lines in Fig. 7, the liquid velocities are always less than the homogeneous velocities. If the measured velocity by the Doppler systems under-estimates the liquid velocity, then it normally under-estimates the homogeneous velocity more. Therefore, in the following sections when serious under- 
estimation occurs, we often only compare the measurements with the reference liquid velocities, knowing that corresponding comparison with the homogeneous velocity is worse.

\section{Velocity measurement of gas/oil/water three-phase flows}

In Fig. 8, the velocities measured by the ultrasonic Doppler are plotted versus the two reference velocities - the liquidphase velocity (left) and the homogeneous velocity (right). In both plots, the velocity measured by ultrasonic Doppler, correlates neither to the reference liquid velocity nor to the homogeneous velocity, except for at $\mathrm{GVF}=0$ (red circles) and a few cases at low GVF values (magenta circles). In general, the ultrasonic Doppler measurements under-estimate the liquid phase velocity. There is a strong connection between worsening under-estimation and rising GVF. This suggests that the underlying cause is a reduced ultrasonic interrogation depth into the flow due to the increased gas bubbles.

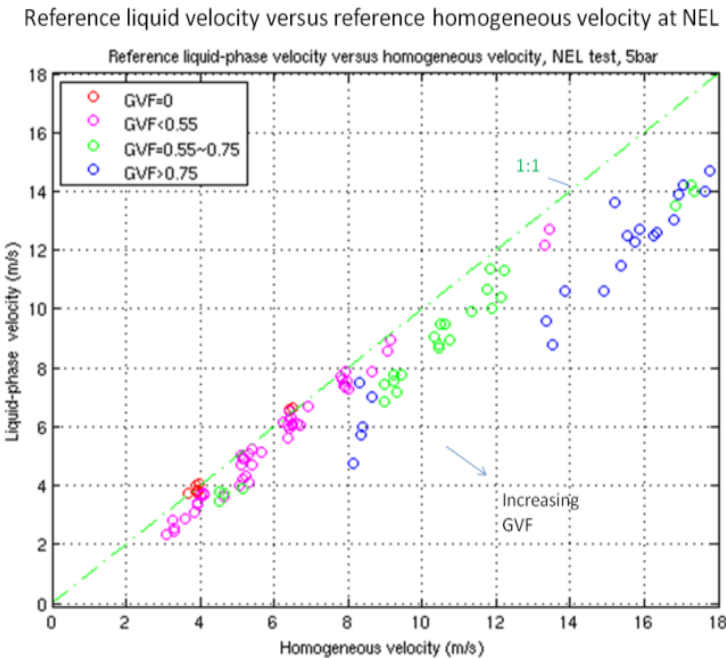

Fig. 7-Reference bulk liquid-phase velocity (derived) versus reference homogeneous velocity for the TUV-NEL test - with different GVF ranges represented by circles of different colors.
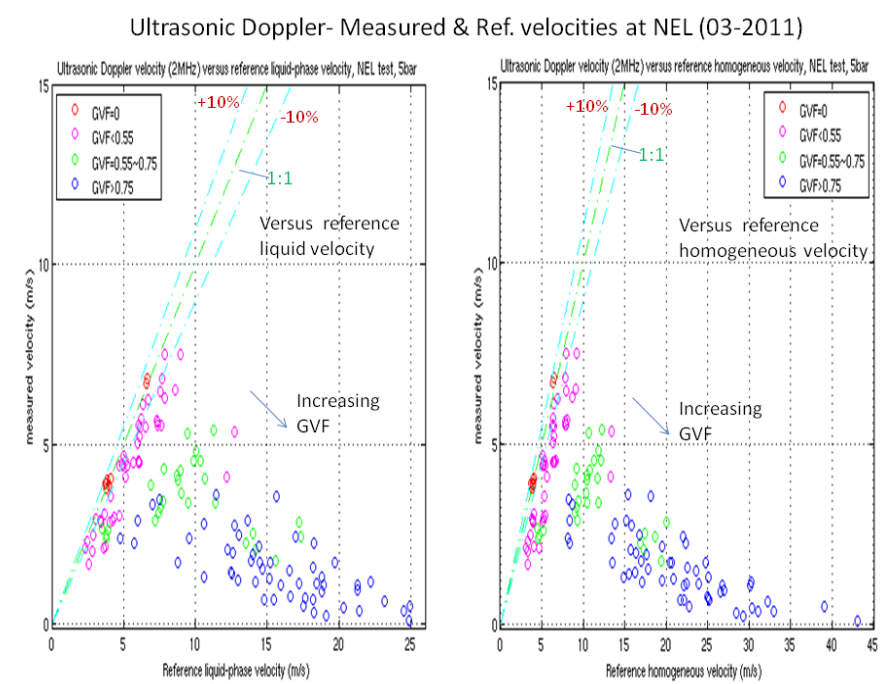

Fig. 8 - Comparing the ultrasonic Doppler velocity measurement with references under gas/oil/water three-phase flow conditions - left plot, measured average Doppler velocities versus reference bulk liquid-phase velocity; right plot, measured versus homogeneous velocity; color of legends shows different ranges of GVF.

A more detailed analysis of the velocity under-estimation is given in Fig. 9, where the relative liquid-phase velocity measurement errors are plotted versus GVF in two subplots. In the left plot, the effect of liquid (volumetric) flow rate, $\mathrm{Q}_{\text {liq }}$, is highlighted by colored legends. In the right plot, the effect of WLR is highlighted. In Fig. 9, a close agreement between the liquid-phase velocity and the velocity measured by the ultrasonic Doppler can only be found at a combination of very low GVF $(<0.25)$ and high WLR $(>0.7)$ values. From middle- to high-GVF values $(\mathrm{GVF}>0.5)$, the effect of liquid flow rate becomes more influential (left plot), showing correlation between increased under-estimation error and rising liquid flow rate, which can be explained by the increased gas bubble concentration in liquid due to the mixing effect of a more turbulent flow. As shown by the plot on the right, WLR has also a significant effect on the measurement errors. The smallest underestimation is associated with WLR $=1$ (magenta circles) where the liquid viscosity is lowest. For GVF $<0.5$, the results become worse as the WLR reduces towards an oil/gas flow, whereas for GVF $>0.5$ and WLR $\leq 0.9$, the effect of WLR is not as strong as the liquid flow rate.

We are not showing error plots for homogeneous velocity because they display trends similar to those in Fig. 9, albeit with worse under-estimation errors (see Fig. 7).

\section{Discussion}

As mentioned in the steps for bulk liquid velocity calculation, an apparent liquid or gas holdup can be derived from an instantaneous Doppler signal-energy versus depth profile, by treating the maximum peak of the profile as the end of the liquid region. The liquid flow velocity is then measured by integrating the Doppler velocity profile from the inner wall of the pipe up to the estimated liquid/gas interface location. For an annular gas/liquid distribution, such an approach should measure the average velocity of the liquid annulus, if the ultrasound wave can propagate up to the interface. For a bubbly distribution, if only low concentration of small-sized bubbles (small slip velocity) exist in the liquid phase, and the ultrasound can travel across the entire pipe, then the liquid flow velocity across the pipe can also be measured. 
Most of the test points in our test matrix are of the slug flow regime, represented by alternation of bubbly and annular distribution sections. In any case, the gas bubble population in liquid has the most important effect on ultrasonic measurement. For low GVF and low liquid flow rate (low Reynolds number) conditions, the gas bubble concentration in the liquid phase is low and ultrasound can propagate sufficiently deep into the liquid phase to make a relatively satisfactory liquid velocity measurement. At higher GVFs (e.g., GVF>0.5), the increased gas bubble population in the liquid phase increasingly limit the interrogation depth of the ultrasound pulse, whose strongest echo is used to locate the end of the liquid region from the pipe wall, whether it is actually from the main gas/liquid interface in an annular distribution or from large gas bubbles inside the liquid layer. The higher the GVF, the shallower is the interrogation depth. The ultrasound Doppler tends to measure the velocity closer to the liquid boundary layer on the inner pipe wall. Note that in an axisymmetric turbulent flow, the velocity profile along a diameter can be approximately divided into a region that is away from the wall with relatively flat velocity distribution and the near-wall regions with large velocity gradients, which are referred to as the boundary layers in this work.

Fig. 10 shows an example from one of the test conditions, where GVF is 0.77 . The time-averaged Doppler signal-energy profile (lower left, which is a histogram of the back-scattered signal energy distribution across the pipe over a period of time) shows that most of the echoes come from the near-wall region. The measured thickness of liquid layer (lower right), underestimates the true thickness by a wide margin, implying the shallow interrogation depth of the ultrasound. The velocity profile (i.e., histogram in the upper left), also shows the dominant contribution of the measured velocities in the near-wall region. The time series of the measured average liquid layer velocity (upper right) shows a close correlation with the measured thickness (lower right); that is, the troughs of the measured velocity always correlate to the troughs of the thickness (smallest interrogation depth), and so do the peaks. Fig. 10 is very different from Figs. 2 and 3, where the corresponding time series and Doppler profiles for a low GVF flow are shown.
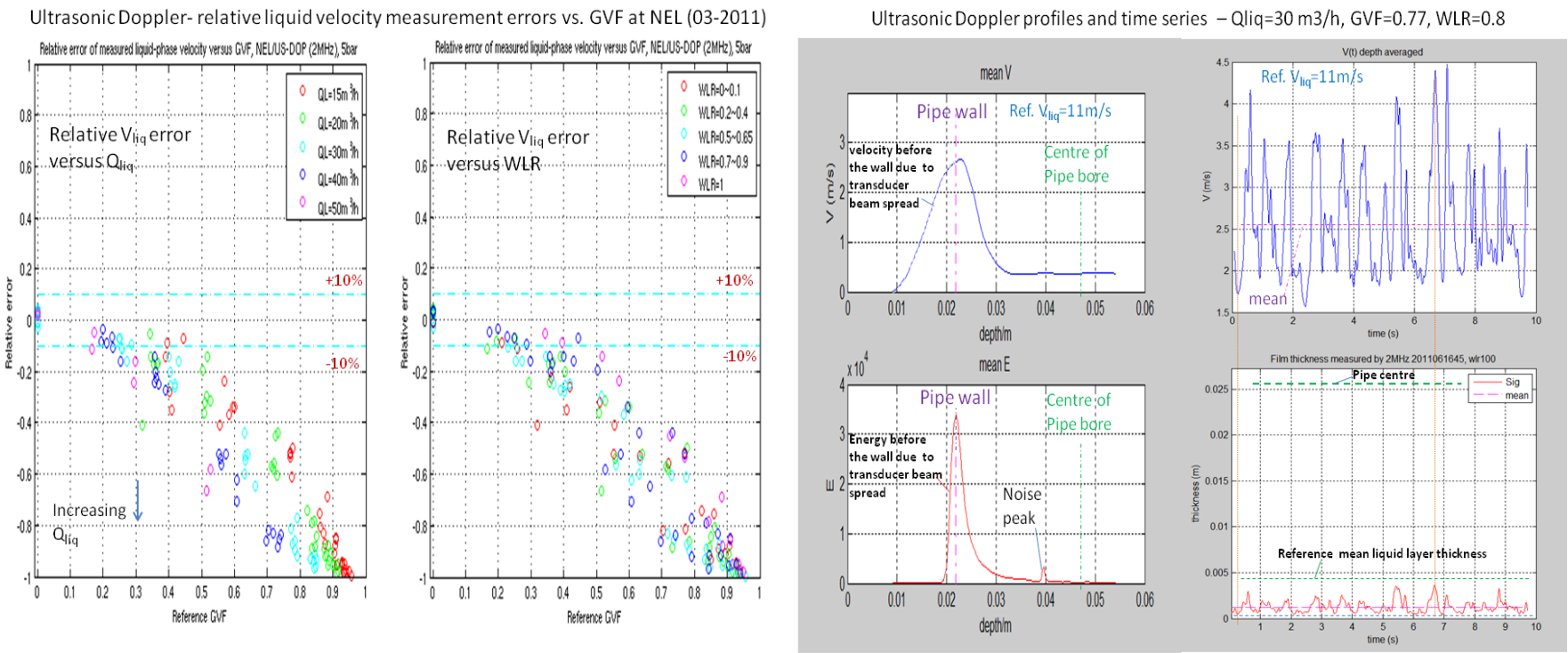

Fig. 9-Relative measurement errors of the ultrasonic Doppler system under gas/oil/water three-phase flow conditions. Liquid-phase velocity measurement errors versus GVF with color of legends showing different ranges of liquidphase flow rate (left); the same error versus GVF plot but with legends showing different ranges of WLR (right).

Fig. 10—Measured ultrasonic Doppler (depth) profiles and time series for a high GVF flow. Time-averaged velocity profile (upper left), timeaveraged Doppler signal-energy profile (lower left), time series of measured bulk liquid velocity through weighted integration from the inner wall to the apparent gas/liquid interface (upper right), time series of measured liquid layer thickness (lower right).

The gas holdup versus the GVF plot shown in Fig. 11 can further explain the issues associated with applications of ultrasonic Doppler measurement to a gas/liquid flow (in this work, vertical upward gas/liquid flows through a Venturi). In Fig. 11, the reference gas holdups are derived from the reference flow rates of the three phases and the measured Venturi $\Delta \mathrm{P}$, following Eq (1). These reference gas holdups are generally situated below the "nonslip" line, indicating that the velocity of the gas phase is greater than that of the liquid phase. The gas holdup values derived from the ultrasonic Doppler measurements are considerably greater than the reference values for the same flow conditions (same color), indicating a restricted ultrasonic interrogation depth into the liquid phase (hence underestimation of liquid holdup). At higher GVF values (e.g., greater than 0.55), some of the measured gas holdup values are close to unity, implying a very shallow ultrasonic interrogation depth and, thus, a velocity measurement region mostly in the boundary layer next to the inner pipe wall. Also, for the same GVF value, the interrogation depth reduces as the liquid flow rate increases, as shown by broken brown 
envelope line in Fig. 11, due to the increased turbulent mixing between the phases, and hence an increased gas bubble concentration entrained in the liquid phase.

\section{Conclusions}

The output of the ultrasonic Doppler velocity measurement system can be correlated to neither the bulk liquid-phase velocity nor the homogeneous velocity of a vertical gas/liquid multiphase flow. The reason is that the ultrasonic interrogation depth is limited by the gas bubble population in the liquid phase, confining the measurement towards the boundary flow layer near the inner pipe wall when the GVF is high, leading to a serious under-estimate of the liquid-phase flow velocity. The pattern of the under-estimation is complex, appearing to depend on the Reynolds number and the properties of the liquids, such as viscosity. Therefore, it is generally difficult to have a robust interpretation of the measured ultrasonic Doppler velocity and to derive the flow rates of a vertical gas/liquid multiphase flow, without resorting to a highly complex empirical model.

\section{Acknowledgement}

We are grateful to Dr. Gary Oddie, Dave Scott, and John Ashworth for their help in the building of the research flow measurement apparatus for testing at TUV-NEL and Schlumberger Gould Research.

\section{References}

[1] Teniou, S. and Meribout, M.: "Multiphase Flowmeters Principles and Applications: A Review Evaluation," Canadian Journal on Scientific and Industrial Research Vol. 2, No. 8, November 2011, pp. 290-293.

[2] Falcone, G., Hewitt, G.F. and Alimonti, C.: "Multiphase Flow Metering: Current Trends and Future Developments," Journal of Petroleum Technology (2002) 54(4) 77-84.

[3] Atkinson, I., Berard, et al.: "A New Horizon in Multiphase Flow Measurement," Oilfield Review, Schlumberger, Winter (2004-2005), 52-63.

[4] Liu, Z. and Liu, Z.R.: "Multiphase Flowmeter Using a Combination of Pressure Differentials and Ultrasound Doppler Readings," US Patent No. 7,963,172 B2, (2011).

[5] Cadalen, S. and Lance, M.: "A Mechanics Approach for Wet Gas Flow Metering, Theory, and Application to Flow Loop Tests," International Journal of Multiphase Flow (2011), 37, No. 3, 260-267.

[6] Morriss, S.L. and Hill, A.D.: "Measurement of Velocity Profiles in Upwards Oil/Water Flow Using Ultrasonic Doppler Velocimetry," paper SPE 22766 presented at the SPE Annual Technical Conference and Exhibition (October 6-9, 1991).

[7] Gardner, D. and Nyhavn, F.: "Production Logging Tool Developments for Horizontal Wells and Hostile Environments,"SPE 36564, October 1996, pp 793-803.

[8] Nyhavn, F., Bang, J. and Oyangen, T.: "Production Logging in Horizontal Wells by Use of Ultrasonics," SPE Production \& Facilities (1999), 14 (3), 161-165.

[9] Evans, D.H. and McDicken, W.N.: Doppler Ultrasound - Physics, Instrumentation and Signal Processing (2nd ed.), John Hoboken, New Jersey, USA, Wiley \& Sons (2000).

[10] Mahadeva, D., Huang, S., Oddie, G. and Baker, R.C.: "Study of the Effect of Beam Spreading on Systematic Doppler Flow Measurement Errors," Proceedings of 2010 IEEE International Ultrasonics Symposium (October 11-14, 2010), San Diego, California, USA, pp711-714.

[11] Huang, S.: "Doppler Flowmeter for Multiphase Flows”, US Patent No. US6,758,100 (2004). 